\title{
Step-down of inhaled corticosteroids in non-eosinophilic asthma: A prospective trial in real life
}

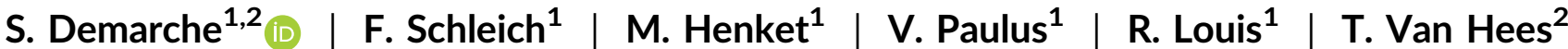

${ }^{1}$ Department of Respiratory Medicine, $\mathrm{CHU}$ Liege, GIGA I ${ }^{3}$ Research Group, University of Liege, Liege, Belgium

${ }^{2}$ Department of Clinical Pharmacy, CIRM (Center for Interdisciplinary Research on Medicines), University of Liege, Liege, Belgium

\section{Correspondence}

Sophie Demarche, Department of Respiratory Medicine, CHU Liege, GIGA I ${ }^{3}$ Research Group, University of Liege, Liege, Belgium.

Email: sophie.demarche@ulg.ac.be

\section{Funding information}

This work was supported by a federal grant IAP ("Interuniversity Attraction Poles"

programme) $\mathrm{P} 7 / 30$.

\section{Summary}

Background: While non-eosinophilic asthmatics are usually considered poorly responsive to inhaled corticosteroids (ICSs), studies assessing a step-down of ICS in this specific population are currently lacking.

Objectives: To assess the proportion of non-eosinophilic asthmatics in whom ICS may be withdrawn without any clinical degradation and to determine the predictive markers of a failure to stop treatment with ICS.

Methods: This prospective study was completed by 36 non-eosinophilic asthmatics, defined by sputum eosinophils $<3 \%$ and blood eosinophils $<400 / \mu \mathrm{L}$. In these patients, whichever the baseline asthma control level, the dose of ICS was gradually reduced every 3 months until they met the failure criteria or successfully discontinued ICS for 6 months. The failure criteria were an ACQ score $\geq 1.5$ with an increase from baseline $>0.5$ or a number of severe exacerbations during the study which was greater than the number during the year prior to the baseline visit. Receiver-operating characteristic (ROC) curves were constructed to assess predictors of a failure to stop ICS. This study is registered with ClinicalTrials.gov, number NCT02169323.

Results: In 14 patients (39\%), ICSs were completely withdrawn, and in 10 further patients (28\%), ICS were stepped-down to a reduced ICS dose without any deterioration of asthma control and exacerbation rate. Baseline predictors of a failure to stop ICS were a greater age (area under ROC curve [ROC AUC] and [95\% Cl]: 0.77 [0.62-0.93]) and elevated blood eosinophils (ROC AUC [95\% CI]: 0.77 [0.61-0.93]). After the first step-down of ICS, the best predictor was an elevated blood eosinophil count (ROC AUC [95\% Cl]: 0.85 [0.72-0.99]).

Conclusions \& Clinical Relevance: Withdrawing or reducing the dose of ICS is feasible in two-thirds of non-eosinophilic asthmatics irrespective of baseline asthma control. An elevated blood eosinophil count may predict the failure to stop ICS.

KEYWORDS

asthma, asthma control, blood, eosinophils, exacerbation, inhaled corticosteroids, noneosinophilic, sputum 


\section{1 | INTRODUCTION}

Asthma is a heterogeneous disease, which can be classified into various phenotypes according to clinical, inflammatory or physiologic characteristics, treatment responsiveness and prognostic factors. ${ }^{1}$ The technique of induced sputum has allowed to define 2 inflammatory phenotypes: eosinophilic and non-eosinophilic asthma. $^{2}$

According to international guidelines, ICSs are the first-line controller treatment in asthmatic patients, ${ }^{3}$ whichever their inflammatory phenotype. Non-eosinophilic asthmatics are, however, usually considered poorly responsive to ICS. ${ }^{4}$ Several studies have indeed shown that the effect of short-term treatment with ICS on symptoms, quality of life, baseline airway calibre and bronchial hyperresponsiveness was lower in non-eosinophilic asthmatics as compared with their eosinophilic counterparts. ${ }^{5-8}$ Whether these non-eosinophilic asthmatics are unresponsive, ${ }^{5,6}$ partly responsive $e^{7,8}$ or well responsive $^{9}$ to ICS is still controversial. This is, however, an important concern because they account for approximately $60 \%$ of corticosteroid-naive asthmatic patients when the threshold to define sputum eosinophilia is set at $3 \%{ }^{10}$

In studies assessing the impact of discontinuing or reducing the dose of ICS in controlled asthmatics, increased sputum eosinophils at baseline ${ }^{11,12}$ or a raise in sputum eosinophils during the stepdown ${ }^{13-15}$ was found to be predictive markers for asthma deterioration. These studies had, however, included both eosinophilic and non-eosinophilic patients. Nevertheless, it has been suggested that stepping-down ICS in eosinophilic asthmatics might put those patients at risk of exacerbations. ${ }^{11}$ A step-down of ICS may, however, be acceptable in non-eosinophilic asthmatics, ${ }^{16}$ but studies in this specific population are currently lacking.

The aims of our study were twofold. First, to assess the proportion of non-eosinophilic patients in whom ICS may be withdrawn without any degradation of asthma control and exacerbation rate and second, to determine the predictive markers of a failure to stop treatment with ICS.

\section{2 | METHODS}

\section{1 | Setting and participants}

Patients were recruited from the University Asthma Clinic of Liege, between 20 June 2014 and 30 June 2016. The diagnosis of asthma was defined by the presence of typical symptoms (wheezing, breathlessness, chest tightness, cough) and at least one of the following criteria: a forced expiratory volume in 1 second $\left(\mathrm{FEV}_{1}\right)$ increase of at least $12 \%$ and $200 \mathrm{~mL}$ after inhalation of $400 \mu \mathrm{g}$ salbutamol and/or a provocative concentration of methacholine causing a $20 \%$ fall in $\mathrm{FEV}_{1}$ less than $16 \mathrm{mg} / \mathrm{mL}$. Patients were eligible for the study if they were asthmatics aged of at least 18 years, had a sputum eosinophil count $<3 \%$ combined with a blood eosinophil count $<400 / \mu \mathrm{L}^{17}$ and were treated with ICS at the same dose since the previous
3 months. Patients were excluded if they had a history of near-fatal asthma requiring a stay in intensive care unit, if they were treated with oral corticosteroids (OCSs) at screening visit or had been treated with OCS in the previous 4 weeks, if they were treated with omalizumab or if they were pregnant women. The study was approved by the Ethics Committee of the University Hospital of Liege (Reference 2014/98) and registered with ClinicalTrials.gov, number NCT02169323. All patients gave written informed consent.

\subsection{Study design}

The study was prospective, monocentric and interventional. The study design is shown in Figure 1.

Before stepping-down ICS, an intermediate phase of treatment step-up was performed at the beginning of the study in patients whose asthma was uncontrolled despite a low or medium dose of ICS. In these asthmatics, the treatment was stepped-up to the GINA step 4 with a high dose of ICS for 3 months. This stage was included in the study design to ensure that each patient was treated in accordance with the international guidelines before stepping-down the ICS dose. This step-up phase also gave us the opportunity to study the impact of an increased dose of ICS in symptomatic non-eosinophilic asthmatics.

For all study patients, the intervention consisted of a progressive step-down of ICS every 3 months according to the dose levels defined by the GINA guidelines (from a high dose to a medium dose to a low dose to a complete cessation) until the patients met the failure criteria (see below) or discontinued ICS for 6 months. The visit preceding the first step-down was considered as the baseline visit. At each 3-month visit or whenever during the study on request by the patient, the ACQ score and the number of severe exacerbations were assessed to determine the pursuit of the study. This assessment was always performed outside an asthma exacerbation. An ACQ score $\geq 1.5$ with an increase from the baseline score by more than 0.5 point and/or a cumulated number of severe exacerbations from baseline greater than the number of severe exacerbations during the year prior to the baseline visit were deemed as failure criteria in the study. Patients meeting at least one of these criteria were therefore withdrawn from the study and were prescribed an adequate dose of ICS. Patients who did not meet the failure criteria continued the study protocol.

As described in Figure 1, patients had a detailed investigation at study entry, at each 3-month visit and at the last visit. If patients had a severe exacerbation at any of these scheduled visits, the detailed investigation was postponed and performed at least 4 weeks after the last dose of OCS.

\section{3 | Study investigations}

Atopy was defined as the presence of 1 or more positive specific IgE $(>0.35 \mathrm{kU} / \mathrm{L})$ to at least one of the following aeroallergens: cat, dog, grass pollen, tree pollen, house dust mite and a mould mixture (Phadia, Groot-Bijgaarden, Belgium). Fractional exhaled nitric oxide 


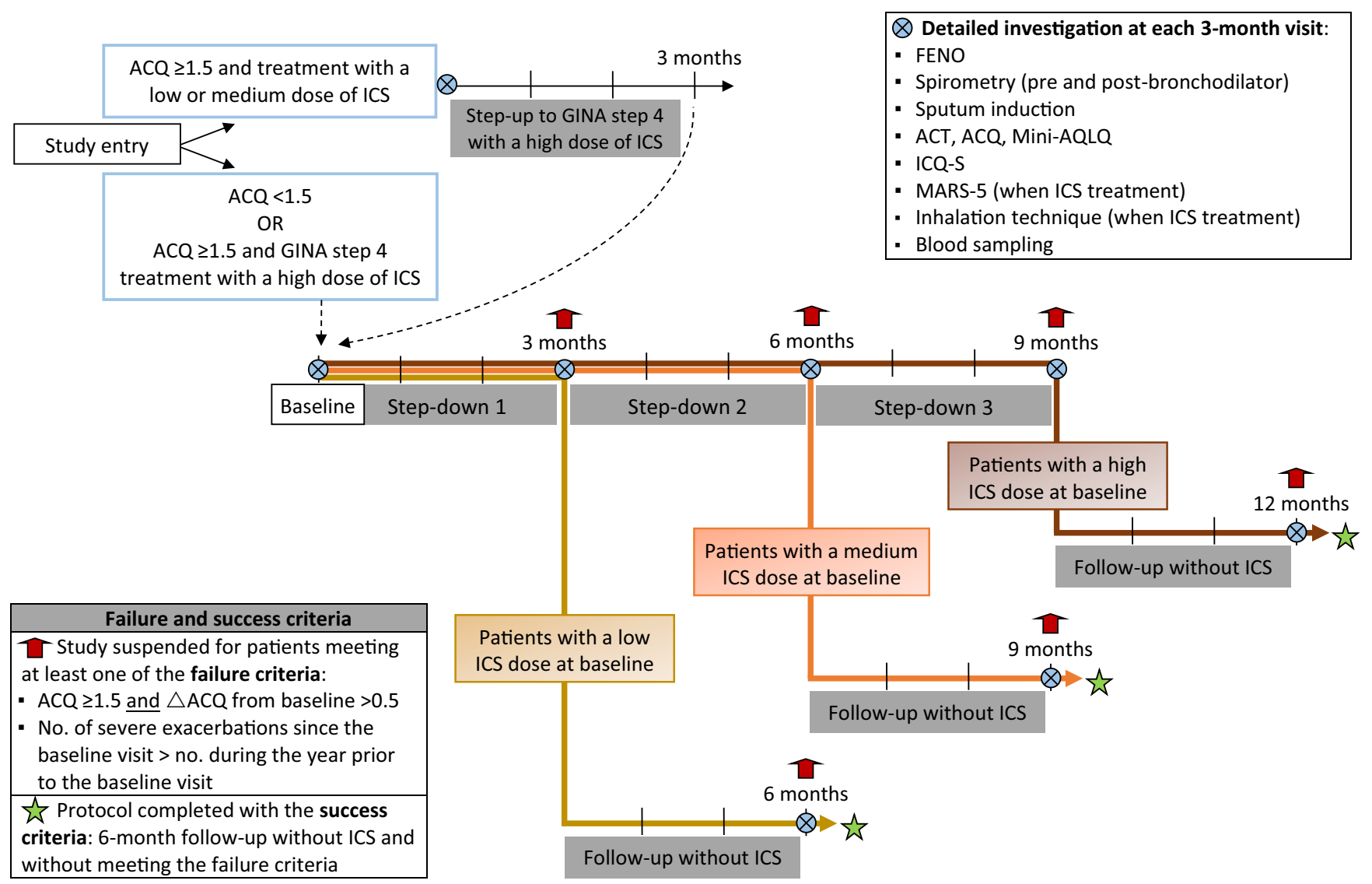

FIGURE 1 Schematic representation of the study design. ACQ, asthma control questionnaire; ACT, asthma control test; AQLQ, asthma quality of life questionnaire; FENO, fractional exhaled nitric oxide; ICQ-S, shortened version of the Inhaled corticosteroids side-effect questionnaire; ICS, inhaled corticosteroid; MARS-5, 5-item medication adherence report scale

(FENO) was measured at a flow rate of $50 \mathrm{~mL} / \mathrm{s}$ (NIOX; Aerocrine, Solna, Sweden). Sputum induction and processing with the wholesample technique was performed as previously described. ${ }^{18}$ The technician who performed the sputum cell count was blind to the clinical status of patients. Asthma control was assessed by the Asthma Control Test $(\mathrm{ACT})^{19}$ and Asthma Control Questionnaire $(A C Q)^{20}$ scores. Asthma-related quality of life was measured by the Mini Asthma Quality of Life Questionnaire (Mini-AQLQ) score. ${ }^{21}$ The Shortened version of the Inhaled Corticosteroids side-effect Questionnaire (ICQ-S) was used to monitor side-effects of ICS. ${ }^{22}$ The ICQ-S score ranges from 0 (no side-effect) to 90 (highest levels of side-effects). ${ }^{22}$ The adherence to ICS was measured by the 5 -item Medication Adherence Report Scale (MARS-5) score. $^{23,24}$ The MARS- 5 score ranges from 5 (the worst) to 25 (the best adherence). ${ }^{23}$ The inhalation technique for ICS was checked at each visit, and the number of errors was recorded. A severe exacerbation was defined as the use of systemic corticosteroids during at least 3 days or a hospitalization/emergency room visit due to asthma with use of systemic corticosteroids. ${ }^{25}$ We recorded the number of severe exacerbations during the year prior to the baseline visit and throughout the study. At the end of the study, the total number of exacerbations recorded after the baseline visit was annualized. The routine laboratory of the University Hospital of Liege performed the blood cell count and the analysis of C-reactive protein (CRP) and fibrinogen.

\subsection{Statistical analysis}

Data were presented as number of observations and percentages for categorical variables and as mean \pm standard deviation or median (interquartile range) for continuous variables. Data from 2 independent groups were compared using a chi-square or Fisher's exact test for categorical variables, a $t$ test for continuous variables compatible with a normal distribution or a Mann-Whitney test for continuous variables incompatible with a normal distribution. To compare 2 visits of the same patients, we used a McNemar test for binary variables, a paired $t$ test for continuous variables when the differences were compatible with a normal distribution or a Wilcoxon matchedpairs signed-rank test for continuous variables when the differences were not compatible with a normal distribution. The ability of variables to predict an improvement after a treatment step-up or to predict a failure to stop ICS was assessed by constructing receiveroperating characteristic (ROC) curves, and optimal thresholds were determined by the method of the nearest point to $(0,1)$. For the variables in each Table, when the number of missing values exceeded $10 \%$, the number of observations was stated in the 
Table caption. A $P$ value $<.05$ was considered statistically significant. Statistical analysis was carried out using STATA version 13.0 (Statistical Software; StataCorp LP, College Station, TX, USA). GraphPad Prism version 7.03 (GraphPad Software, La Jolla, CA, USA) was used for Figure 3.

\section{3 | RESULTS}

A total of 312 asthmatic patients were assessed for eligibility, of whom 43 were included in the study and 36 completed the protocol (Figure 2). From these patients, 14 were successfully weaned off ICS, while 22 patients failed to stop ICS. The median time of followup was 9 months (IQR: 5-13 months).

\section{1 | Treatment step-up in uncontrolled patients}

At study entry, 13 patients had an ACQ score $\geq 1.5$ while not being treated with a high dose of ICS. Overall in these patients, a treatment step-up to the GINA step 4 with high-dose ICS did not result in any functional, clinical or inflammatory changes, apart from a reduction in FENO $(P=.02$, Table 1$)$. We, however, noticed that 5 patients had a clinically significant improvement in asthma control after this treatment step-up (defined as a decrease in ACQ $>0.5$ and/or an increase in ACT >3). These 5 patients were compared with the remaining 8 patients regarding their characteristics at study entry. Those with a significant improvement in asthma control had a lower ACT score (mean $\pm \mathrm{SD}$ : $13 \pm 3$ vs $17 \pm 3, P=.03$ ) and a higher FENO (median [IQR]: 34 [19-57] vs 13 [10-18] ppb, $P=.04$ ) at study entry than patients without any improvement in asthma control after the treatment step-up. The area under the ROC curve (ROC AUC) to identify patients who benefited from a treatment step-up was 0.86 for ACT (95\% Cl: 0.66-1.00), the best threshold being $<16$ points (sensitivity: $80 \%$, specificity: $75 \%$, positive predictive value [PPV]: $67 \%$, negative predictive value [NPV]: $86 \%$, $\mathrm{N}=13$ ). The ROC AUC for FENO was 0.88 (95\% Cl: $0.66-1.00)$, the best threshold being $>20 \mathrm{ppb}$ (sensitivity: $75 \%$, specificity: $88 \%$, PPV: $75 \%$, NPV: $88 \%, \mathrm{~N}=12$ ).

\subsection{Step-down of ICS}

Demographic characteristics of all study patients are summarized in Table 2. Functional, clinical, inflammatory and treatment characteristics at baseline, after the first step-down, and at the last visit in

Adult asthmatics assessed for eligibility $(\mathrm{N}=312)$

\section{Excluded ( $\mathrm{N}=\mathbf{2 6 9})$}

- Not meeting inclusion criteria/meeting exclusion criteria $(N=267)$

- Unsuccessful sputum induction/analysis ( $N=55)$

- Sputum eosinophils $\geq 3 \%$ ( $N=119$ )

- Blood eosinophils $\geq 400 / \mu \mathrm{L}(\mathrm{N}=11)$

- Untreated with ICS ( $N=65)$

- Change in the ICS dose in the previous 3 months $(\mathrm{N}=3)$

- Previous stay in ICU for asthma $(\mathrm{N}=2)$

- OCS in the 4 weeks prior to the visit ( $N=12)$

- Declined to participate $(\mathrm{N}=2)$

Included ( $\mathbf{N}=\mathbf{4 3})$

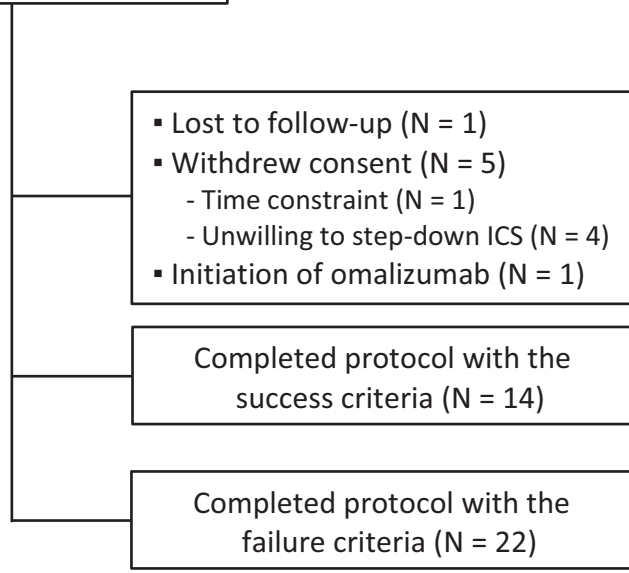

FIGURE 2 Flow diagram (adapted from the CONSORT flow diagram). ICS, inhaled corticosteroid; ICU, intensive care unit; OCS, oral corticosteroid 
TABLE 1 Patient characteristics and effect of treatment step-up in uncontrolled non-eosinophilic asthmatics treated with a low or medium dose of ICS at study entry

Non-eosinophilic asthmatics with an ACQ $\geq 1.5$ and treated with a low or medium dose of ICS at study entry $(\mathrm{N}=13)$

\begin{tabular}{|c|c|c|c|}
\hline Age, years & \multicolumn{2}{|c|}{$54(50-65)$} & \\
\hline Women, N (\%) & \multicolumn{2}{|c|}{$7(54)$} & \\
\hline Atopy, N (\%) & \multicolumn{2}{|c|}{$4(31)$} & \\
\hline $\mathrm{BMI}, \mathrm{kg} / \mathrm{m}^{2}$ & \multicolumn{2}{|c|}{$28.8 \pm 5.7$} & \\
\hline \multicolumn{4}{|l|}{ Smoking status, N (\%) } \\
\hline \multirow[t]{2}{*}{ Ex-smokers } & \multicolumn{2}{|c|}{$6(46)$} & \\
\hline & \multicolumn{2}{|l|}{ Visit before step-up } & $P$ value \\
\hline $\mathrm{FEV}_{1}, \%$ predicted & \multicolumn{2}{|l|}{$81.5 \pm 15.7$} & .70 \\
\hline FVC, \% predicted & \multicolumn{2}{|l|}{$86.4 \pm 16.0$} & .28 \\
\hline ACQ & $2.4 \pm 0.7$ & $2.2 \pm 0.8$ & .17 \\
\hline Mini-AQLQ & $4.0 \pm 0.8$ & $4.3 \pm 0.9$ & .40 \\
\hline FENO, ppb & $17(12-33)$ & $13(6-20)$ & .02 \\
\hline Sputum eosinophils, $\%^{\mathrm{a}}$ & $0.6(0.2-1.0)$ & $0.6(0.2-2.0)$ & .68 \\
\hline Sputum eosinophils, $\times 10^{3} / \mathrm{g}^{\mathrm{a}}$ & $6(2-18)$ & $12(3-32)$ & .22 \\
\hline Sputum neutrophils, $\%^{a}$ & $77(65-90)$ & $73(47-79)$ & .13 \\
\hline Sputum neutrophils, $\times 10^{3} / \mathrm{g}^{\mathrm{a}}$ & $1444(971-1627)$ & $1385(314-3543)$ & .96 \\
\hline Blood eosinophils, $/ \mu \mathrm{L}$ & 109 (77-178) & $124(78-172)$ & .31 \\
\hline Blood neutrophils, $/ \mu \mathrm{L}$ & 3911 (2611-4838) & $4403(3038-5159)$ & .13 \\
\hline Theophylline, N (\%) & $1(8)$ & $1(8)$ & 1.0 \\
\hline$I_{C Q}-S^{b}$ & $34(25-43)$ & $31(28-44)$ & .95 \\
\hline
\end{tabular}

$\mathrm{ACQ}$, asthma control questionnaire; ACT, asthma control test; AQLQ, asthma quality of life questionnaire; BDP CFC, beclomethasone dipropionate-chlorofluorocarbon; BMI, body mass index; CRP, C-reactive protein; FENO, fractional exhaled nitric oxide; FEV ${ }_{1}$, forced expiratory volume in 1 second; FVC, forced vital capacity; ICQ-S, shortened version of the inhaled corticosteroids side-effect questionnaire; ICS, inhaled corticosteroid; LABA, long-acting $\beta 2$ agonist; LTRA, leukotriene receptor antagonist.

${ }^{\text {aD }}$ Data available for both visits in 10 patients.

${ }^{b}$ Data available for both visits in 9 patients.

patients with a successful and unsuccessful withdrawal of ICS are presented in Table 3.

At baseline, patients who subsequently failed to be weaned off ICS were older and had higher blood eosinophils than patients who successfully stopped ICS ( $P=.006$ for both comparisons; Table 2 and Table 3, respectively).

A successful withdrawal of ICS ( $N=14)$ was concomitant with an improvement in ACT, a reduced rate of severe exacerbations and a decreased number of blood neutrophils as compared with the baseline visit (Table 3). In this subgroup of 14 patients, 5 (36\%) had a clinically significant improvement in asthma control (defined as a decrease in ACQ $>0.5$ and/or an increase in ACT >3) when ICS treatment was withdrawn. As compared with the 9 other patients of this subgroup, those with an improved asthma control when ICSs were stopped had a higher baseline reversibility (median [IQR]: 6 [58] vs $3[1-4] \%, P=.03$ ), a lower baseline ACT score (mean $\pm \mathrm{SD}$ : $14 \pm 4$ vs $20 \pm 4, \quad P=.01), \quad$ a higher baseline ACQ score (mean $\pm \mathrm{SD}: 2.6 \pm 0.9$ vs $0.8 \pm 0.8, P=.003$ ), a lower baseline 
TABLE 2 Demographic characteristics of all study patients and comparison between patients with a successful and unsuccessful withdrawal of ICS

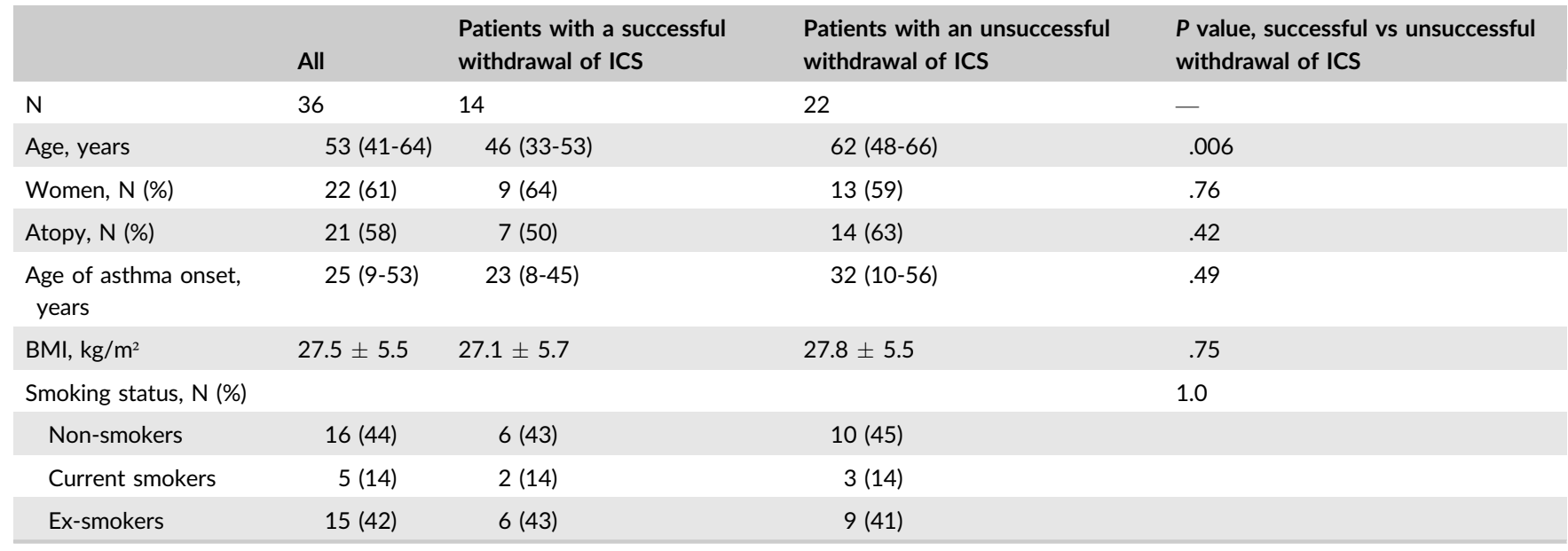

BMI, body mass index; ICS, inhaled corticosteroid.

mini-AQLQ score (mean \pm SD: $3.8 \pm 0.8$ vs $6.0 \pm 0.9, P=.0008$ ), a trend towards higher baseline blood neutrophils (median [IQR]: 5159 [4482-5841] vs $3938[2957-4212] / \mu \mathrm{L}, P=.053)$ and a trend towards higher dose of ICS at baseline (median [IQR]: 2000 [16002000] vs 1000 [400-1000] BDP equivalents, $P=.058$ ). Among the 9 patients whose asthma control did not improve when ICSs were stopped, it should be noted that 3 patients had sputum eosinophils $\geq 3 \%$ at least once during the study.

In patients who failed to stop ICS $(\mathrm{N}=22)$, their last visit was associated with a worsening in $\mathrm{FEV}_{1}$, forced vital capacity (FVC), reversibility to salbutamol, $\mathrm{ACQ}$, sputum eosinophils and blood fibrinogen as compared with baseline (Table 3). In those patients in whom a complete cessation of ICS was unsuccessful, a step-down to a reduced ICS dose was, however, possible without meeting the failure criteria in 10 patients (45\%). In this subgroup, the ICS dose was reduced by a median (IQR) of $1000(400-1600) \mu \mathrm{g}$ beclomethasone CFC equivalents. It is also worth noting that all patients who had an improvement in asthma control after the treatment step-up and who completed the protocol failed to completely stop ICS.

When looking at the evolution of clinical and inflammatory outcomes throughout the study, the loss of asthma control was concomitant with increased blood and sputum eosinophils in patients who failed to stop of ICS, whereas these inflammatory parameters were rather stable over time in patients who successfully discontinued ICS (Figure 3).

In a subgroup analysis, we assessed the success rate of ICS cessation according to the level of asthma control at baseline. In the subgroup of patients with an ACQ $<1.5$ at baseline, ICSs were successfully withdrawn in $40 \%$ of patients ( 8 of 20 ), while the success rate was $38 \%$ (6 of 16 ) in the subgroup of patients with an ACQ $\geq 1.5$ at baseline despite treatment with high-dose ICS.

We also analysed the success rate of ICS cessation according to the season at last visit of patients. The success rate was $50 \%$ (4 of 8 ) in patients whose last study visit was in summer, $45 \%$ (5 of 11) in patients with a last visit in spring, 14\% (1 of 7) in patients with a last visit in autumn and $40 \%$ ( 4 of 10 ) in patients who finished the study in winter ( $P=.54$ for the comparison).

\section{3 | Predictors of a failure to stop ICS}

ROC analyses for predictors of an unsuccessful withdrawal of ICS are presented in Table 4. Predictors at baseline and after the first step-down correspond to the variables that were statistically different between the success and failure groups at these time-points. At baseline, a greater age and elevated blood eosinophils were found to be predictive of a failure to stop ICS (area under ROC curve: 0.77 for both predictors, Table 4). After the first step-down, an elevated number of blood eosinophils were the best predictor of an unsuccessful withdrawal of ICS (area under ROC curve: 0.85, Table 4). A subanalysis of the failure rate of ICS withdrawal according to several thresholds of blood eosinophils at baseline and after the first stepdown was also performed and is shown in Table 5. To find individual predictors of a failure to stop ICS, we compared the absolute changes in the continuous variables of Table 3 from baseline to first step-down between both groups of patients. The variation in the ACQ score was the only variable that was statistically significant between both groups of patients, and its ability to predict an unsuccessful withdrawal of ICS is presented in Table 4 (area under ROC curve: 0.76).

In the subgroup of patients who had an ACQ $\geq 1.5$ despite treatment with high-dose ICS before the step-down, predictors at baseline of a failure to stop ICS were a greater age and elevated blood eosinophils, while the only predictor after the first step-down was an elevated blood eosinophil count (Table 6).

\subsection{Adherence to ICS and inhalation technique}

At each visit (except when patients were weaned off ICS), the adherence and inhalation technique were measured for ICS. Adherence to ICS was good in both groups at baseline (median MARS-5 


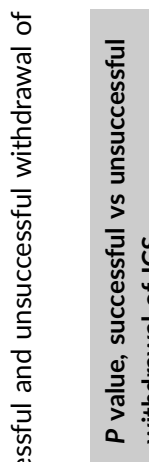

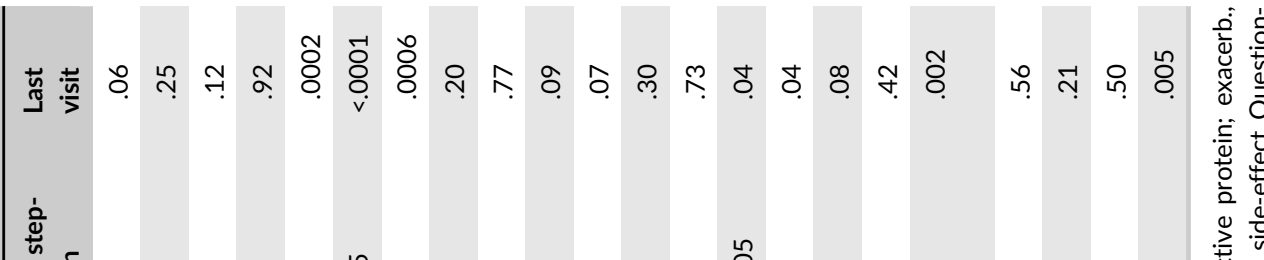

节

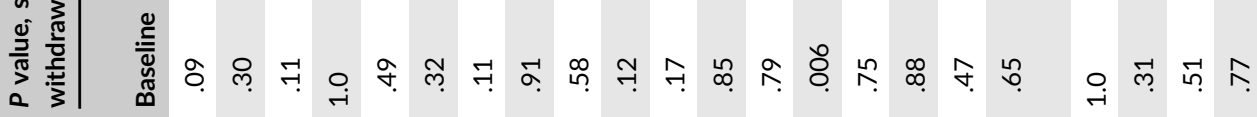

$\breve{\breve{s}}$

$\stackrel{\text { 告 }}{3}$

莺

중

$\stackrel{5}{: \frac{5}{5}}$

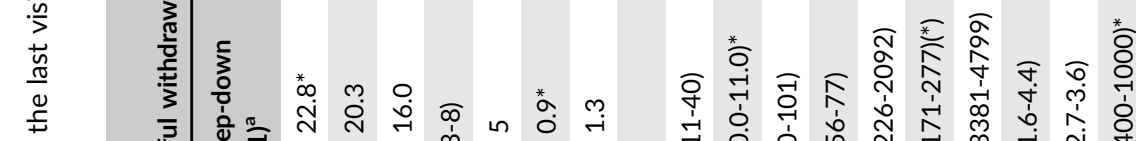

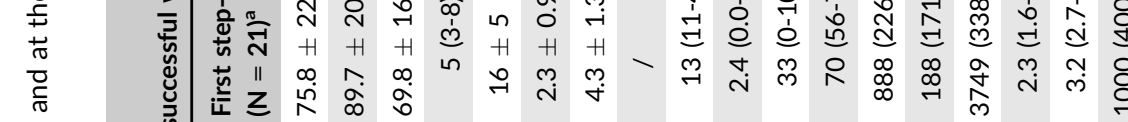

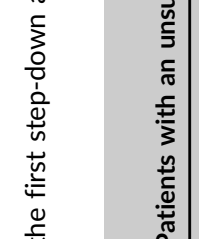

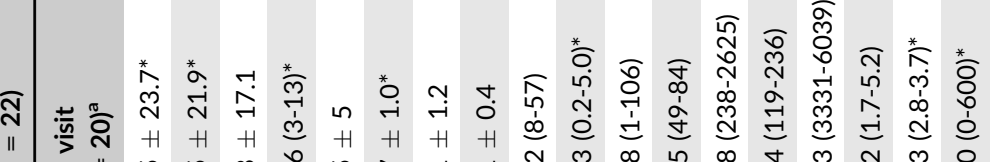

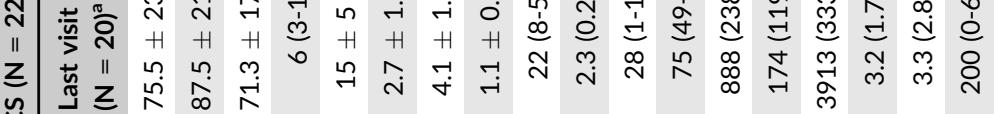

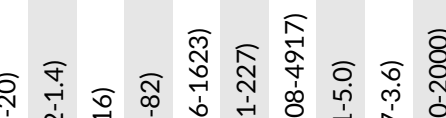

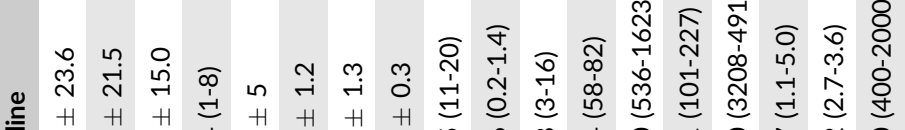

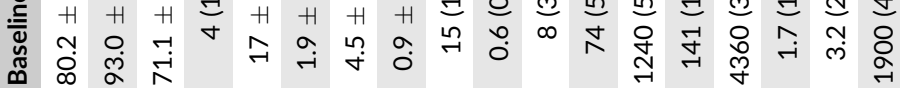

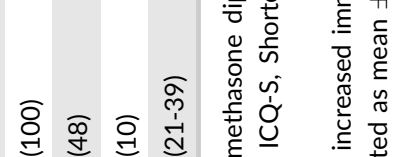

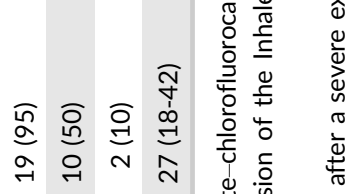

त 워

을

ญ

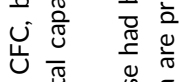

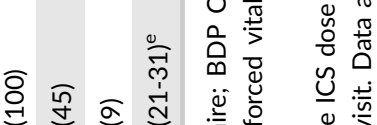

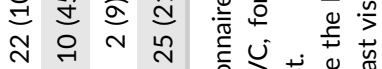

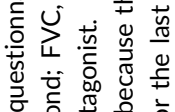

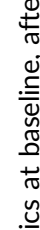

.$\stackrel{n}{\frac{n}{n}}$

$\frac{\mathbb{E}}{\frac{\pi}{\pi}}$

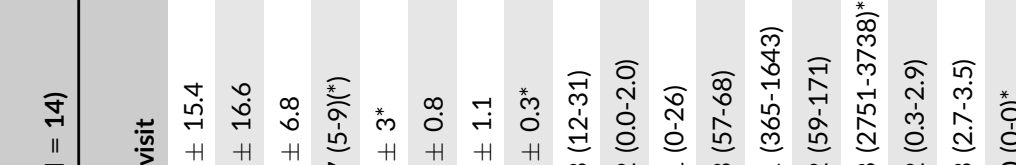

苞

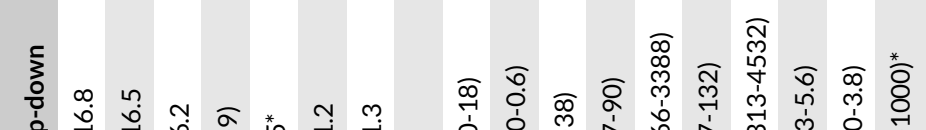

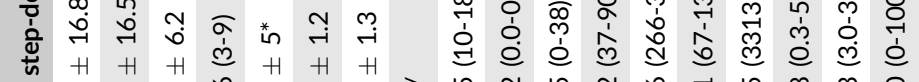

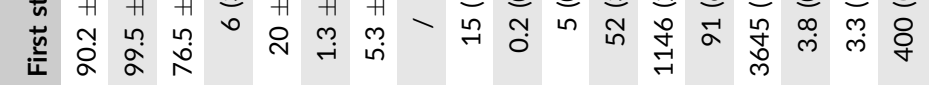

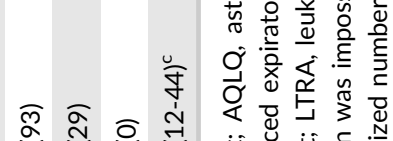

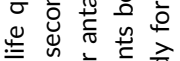

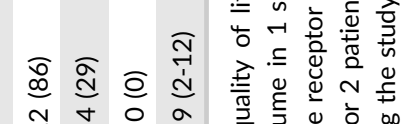

I

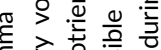

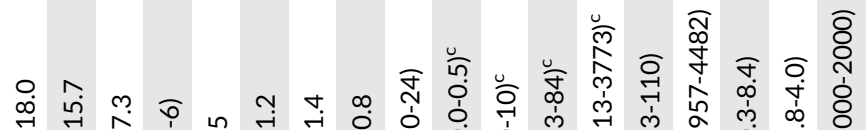

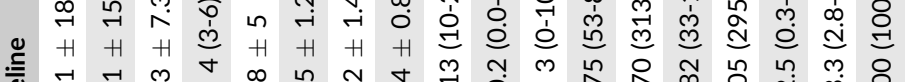

$\stackrel{\frac{\pi}{\underline{L}}}{.}$

产

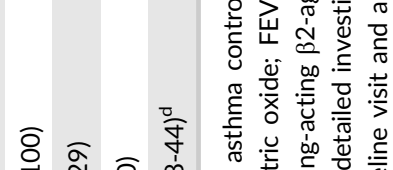

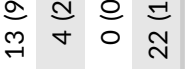

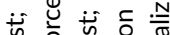

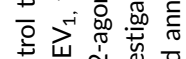

t

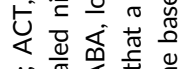

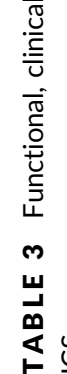

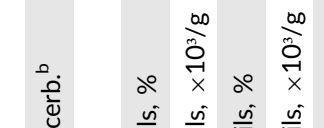

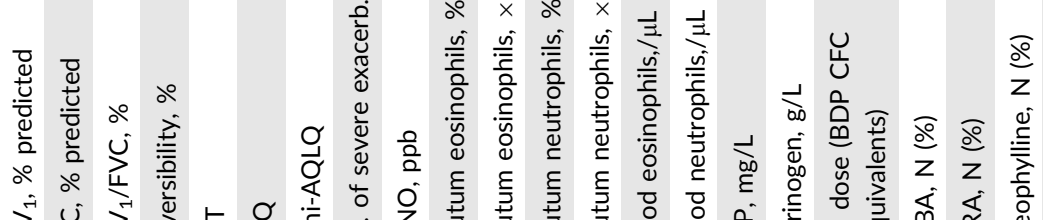

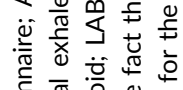

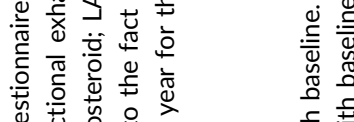

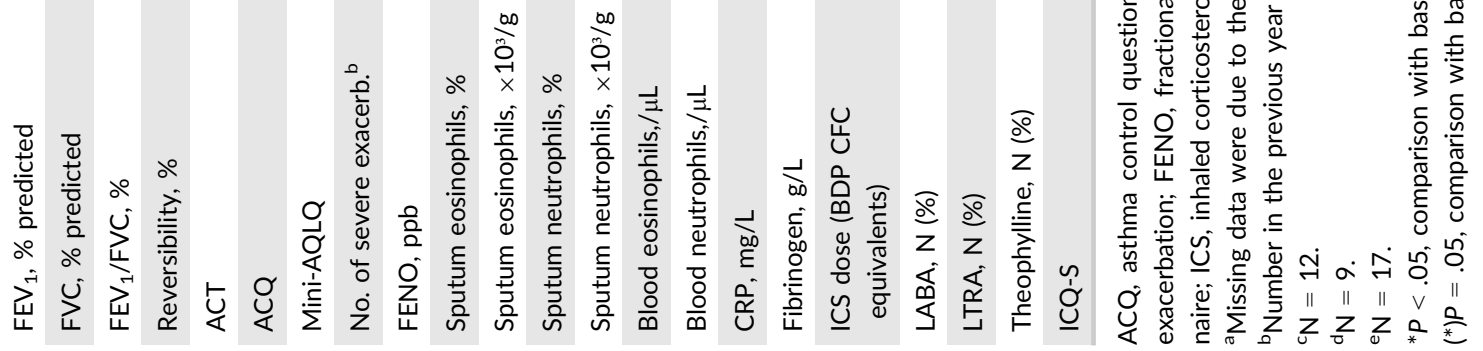




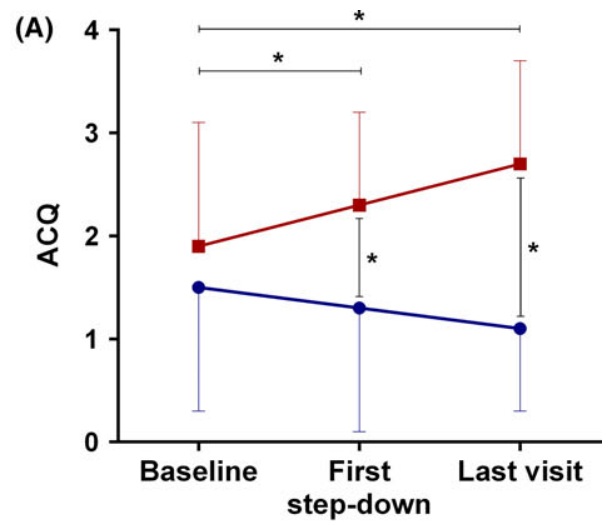

(B)
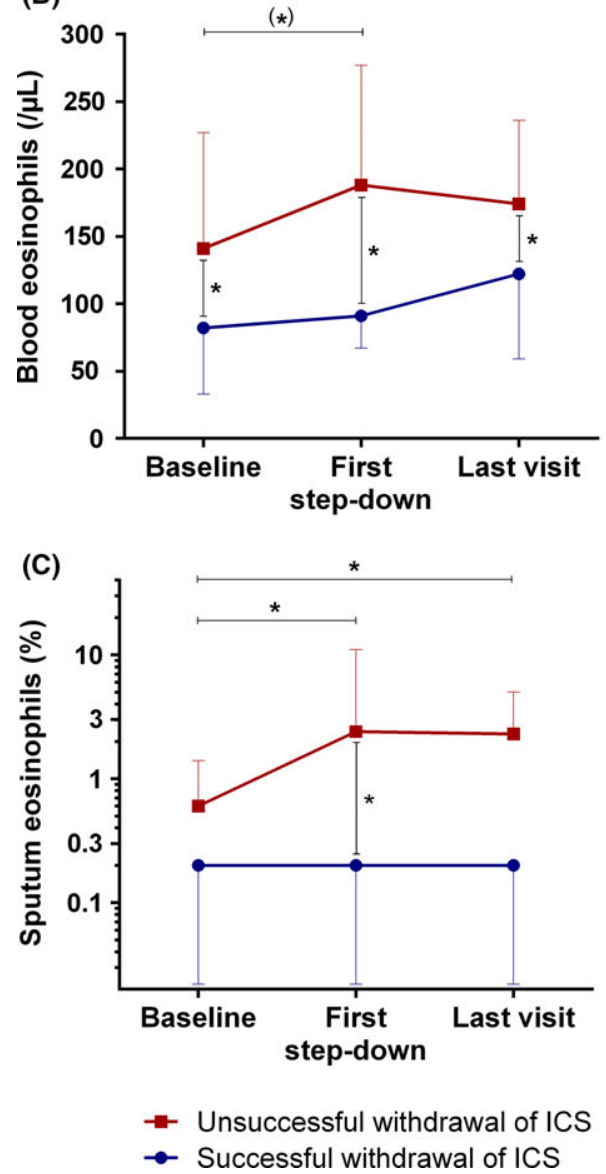

FIGURE 3 ACQ score (A), absolute blood eosinophil count (B) and sputum eosinophil percentage $(C)$ at baseline, after the first step-down and at the last visit in patients with a successful and unsuccessful withdrawal of ICS. Data are expressed as mean (SD) for ACQ and median (IQR) for blood and sputum eosinophils. * $P<.05$, (*) $P=.05$. ACQ, asthma control questionnaire; ICS, inhaled corticosteroid

score [IQR]: 24 [21-25] in the success group and 25 [23-25] in the failure group) and at subsequent visits (median MARS-5 score [IQR]: 24 [23-25] in the success group and 25 [24-25] in the failure group). The median (IQR) number of errors in the inhalation technique was 1 (0-2) for both groups at baseline and was $0(0-1)$ in the success group and $1(0-1)$ in the failure group at subsequent visits.

\section{DISCUSSION}

The importance of personalized medicine has been largely emphasized over recent years. Corticosteroid responsiveness in asthma is certainly a major issue in this regard. In corticosteroid-naive patients, the presence of eosinophilic airway inflammation seems necessary to have a good clinical response to ICS. Our study shows that stepping-down ICS in non-eosinophilic asthmatics is feasible in clinical practice irrespective of baseline asthma control. About one-third of the patients can be completely weaned off ICS while 1 further third can be stepped-down to a reduced ICS dose without compromising asthma control and exacerbation rate. In those patients who are getting uncontrolled when withdrawing ICS, it is of interest that blood eosinophil count may alert the clinician to the risk of asthma deterioration.

Although the major aim of our study was to investigate the stepdown of ICS in non-eosinophilic asthmatics, an intermediate phase of step-up was included in our protocol for those patients who were uncontrolled at study entry despite a treatment with a low or moderate dose ICS. While this step-up did not result in any overall clinical improvement when the whole subgroup was analysed, it is noteworthy that those individual patients with higher FENO levels had a clinically significant improvement in asthma control (defined as a decrease in ACQ $>0.5$ and/or an increase in ACT $>3)^{3}$ with an increased dose of ICS. These data are in keeping with several studies showing that some steroid-naive non-eosinophilic patients might benefit from ICS, ${ }^{8,9,26}$ particularly those with increased FENO levels. $^{8}$

It has been suggested that sputum ${ }^{11-15}$ and blood ${ }^{15}$ eosinophils may predict asthma deterioration following a decrease or withdrawal of ICS in well-controlled asthmatics. In non-eosinophilic patients defined by sputum eosinophils $<3 \%$ and blood eosinophils $<400 / \mu \mathrm{L}$, our data show that elevated blood eosinophils at baseline and elevated blood or sputum eosinophils after the first step-down phase were predictive of a failure to withdraw ICS. As previously suggested, ${ }^{27}$ a low-grade eosinophilic inflammation may therefore exist in non-eosinophilic asthmatics and may explain an unsuccessful withdrawal of ICS. As far as FENO is concerned, it did not predict a failure to stop ICS in our study, in accordance with previous findings. ${ }^{13,14}$

International guidelines recommend to step-down treatment once asthma control has been achieved for at least 3 months. ${ }^{3}$ However, in keeping with studies tailoring asthma treatment according to sputum eosinophils, ${ }^{16,28}$ we chose to step-down ICS in non-eosinophilic asthmatics irrespective of baseline asthma control. It is therefore worth noting that one-third of uncontrolled asthmatics despite treatment with high-dose ICS at baseline were successfully weaned off ICS without any clinical degradation. Besides, monitoring sputum eosinophils during the step-down process is of importance because some patients may tolerate an increase in sputum eosinophils without any degradation in asthma control, as previously reported. ${ }^{18,29}$ In those patients, the ICS dose should be raised to reduce the risk of exacerbations associated with eosinophilic inflammation. ${ }^{30,31}$ 
TABLE 4 ROC analysis for predictors of a failure to stop ICS

\begin{tabular}{|c|c|c|c|c|c|c|c|c|}
\hline & $\mathrm{N}$ & AUC & $95 \% \mathrm{Cl}$ & Best threshold & Sensibility & Specificity & PPV & NPV \\
\hline \multicolumn{9}{|l|}{ Predictors at baseline } \\
\hline Age, years & 36 & 0.77 & $0.62-0.93$ & $>59$ & $59 \%$ & $93 \%$ & $93 \%$ & $59 \%$ \\
\hline \multicolumn{9}{|c|}{ Predictors after the first step-down } \\
\hline ACQ & 35 & 0.79 & $0.61-0.96$ & $>1.5$ & $86 \%$ & $71 \%$ & $82 \%$ & $77 \%$ \\
\hline Sputum eosinophils, \% & 34 & 0.73 & $0.56-0.90$ & $>0.9 \%$ & $65 \%$ & $86 \%$ & $87 \%$ & $63 \%$ \\
\hline Blood eosinophils, $/ \mu \mathrm{L}$ & 35 & 0.85 & $0.72-0.99$ & $>141$ & $81 \%$ & $86 \%$ & $89 \%$ & $75 \%$ \\
\hline \multicolumn{9}{|c|}{ Predictors based on the change from baseline to the first step-down } \\
\hline Change in $\mathrm{ACQ}$ & 35 & 0.76 & $0.60-0.92$ & $>0.2$ & $57 \%$ & $93 \%$ & $92 \%$ & $59 \%$ \\
\hline
\end{tabular}

ACQ, asthma control questionnaire; ACT, asthma control test; AQLQ, asthma quality of life questionnaire; AUC, area under the curve; NPV, negative predictive value; PPV, positive predictive value.

TABLE 5 Failure rate of ICS withdrawal according to blood eosinophil levels

\begin{tabular}{|lcc|}
\hline & N & $\begin{array}{l}\text { Patients with an } \\
\text { unsuccessful withdrawal of ICS, N (\%) }\end{array}$ \\
\hline Blood eosinophils at baseline & 14 & $5(36)$ \\
\hline Blood eosinophils $<100 / \mu \mathrm{L}$ & 24 & $12(50)$ \\
\hline Blood eosinophils $<150 / \mu \mathrm{L}$ & 28 & $15(54)$ \\
\hline Blood eosinophils $<200 / \mu \mathrm{L}$ & 34 & $20(59)$ \\
\hline Blood eosinophils $<300 / \mu \mathrm{L}$ & 36 & $22(61)$ \\
\hline Blood eosinophils $<400 / \mu \mathrm{L}$ & 10 & $2(20)$ \\
\hline Blood eosinophils after the first step-down & $5(29)$ \\
\hline Blood eosinophils $<100 / \mu \mathrm{L}$ & 17 & $13(50)$ \\
\hline Blood eosinophils $<150 / \mu \mathrm{L}$ & 26 & $17(55)$ \\
\hline Blood eosinophils $<200 / \mu \mathrm{L}$ & 31 & $19(58)$ \\
\hline Blood eosinophils $<300 / \mu \mathrm{L}$ & 33 & \\
\hline Blood eosinophils $<400 / \mu \mathrm{L}$ & & \\
\hline
\end{tabular}

TABLE 6 ROC analysis for predictors of a failure to stop ICS in patients with a baseline ACQ $\geq 1.5$ despite treatment with high-dose ICS

\begin{tabular}{|c|c|c|c|c|c|c|c|c|}
\hline & $\mathrm{N}$ & AUC & $95 \% \mathrm{Cl}$ & Best threshold & Sensibility & Specificity & PPV & NPV \\
\hline \multicolumn{9}{|l|}{ Predictors at baseline } \\
\hline Age, years & 16 & 0.91 & $0.77-1.00$ & $>53$ & $80 \%$ & $83 \%$ & $89 \%$ & $71 \%$ \\
\hline \multicolumn{9}{|c|}{ Predictors after the first step-down } \\
\hline Blood eosinophils, $/ \mu \mathrm{L}$ & 16 & 0.83 & $0.62-1.00$ & $>108$ & $80 \%$ & $83 \%$ & $89 \%$ & $71 \%$ \\
\hline
\end{tabular}

AUC, area under the curve; ICS, inhaled corticosteroids; NPV, negative predictive value; PPV, positive predictive value.

Several systematic reviews and meta-analyses of randomized controlled trials assessing the step-down of ICS have been published so far. Rank et $\mathrm{al}^{32}$ reported that withdrawing low-dose ICS was associated with a higher risk of exacerbations, which occurred in $38 \%$ of those patients over a mean period of 6 months. In contrast, Hagan et $\mathrm{al}^{33}$ suggested that reducing ICS to a lower dose did not increase the risk of asthma exacerbations while in a recent article,
Crossingham et $\mathrm{al}^{34}$ concluded that the body of evidence was insufficient to draw conclusions about the risks and benefits of steppingdown ICS. In these studies, the prevalence of exacerbations was $21 \%$ and $18 \%$, respectively, over a mean follow-up of 5-6 month in patients whose ICSs were reduced. These results are, however, not comparable with our data, where we found that stepping-down ICS was unsuccessful in $33 \%$ of patients, as the failure criteria in our 
study included both exacerbations and worsening in asthma control. Moreover, in studies included in these meta-analyses, patients were selected based on a good asthma control but not according to their eosinophilic phenotype. However, we are convinced that the stepdown approach in these studies would have been more effective if sputum eosinophils had been taken into account.

Remarkably, in our study, an improvement in asthma control and a reduction in exacerbation rate were observed in patients who were successfully weaned off ICS. The reasons for this improvement are unclear, but one can hypothesize that local side-effects and reduced local immunity with ICS might play a role. Although withdrawing ICS was not possible in $60 \%$ of the study population, a reduction in the ICS dose was feasible in half of these patients without clinical degradation over a 3-month period. In clinical practice, the importance to find the lowest effective treatment with ICS is explained by the fact that high-dose ICSs are associated with local and systemic sideeffects, ${ }^{35,36}$ increased risk of respiratory infections ${ }^{37,38}$ and costs.

A strength of our study is that there are currently little data in the literature on ICS step-down in non-eosinophilic asthmatics. Moreover, the time of follow-up was reasonably long in our protocol, with step-down phases every 3 months and an observation time of patients without ICS of 6 months.

Our study has some limitations. First, non-eosinophilic asthmatics were defined based on a single measurement of sputum and blood eosinophils at baseline. Therefore, we cannot exclude that a part of the study patients had intermittently eosinophilic asthma, a phenotype known to partly respond to corticosteroids. ${ }^{39}$ Second, because all patients defined as non-eosinophilic at study entry were treated with ICS when sputum and blood were sampled, we acknowledge that a part of our study population was probably misclassified as non-eosinophilic. This was confirmed by the fact that some patients showed a recrudescence of eosinophilic inflammation when ICSs were stepped-down. A way to correctly assess the inflammatory phenotype of patients would have been to measure sputum and blood eosinophils during a steroid withdrawal step at study entry, but this was impracticable in a real-life clinical trial. Third, our study had no control group, which means that we could not compare our results with those obtained in a group of patients who were kept on a stable dose of ICS. Fourth, our study was not blinded and we may hypothesize that the failure rate would have been lower in a blinded study, which may be explained by the presence of some patients anxious to be deprived of a chronic treatment supposed to be essential for controlling their disease. Fifth, our study was not powered to assess the impact of seasonal changes on the success rate of the stepdown process, but we can hypothesize that such changes might contribute to the failure to stop ICS in some patients. Finally, as it was a monocentric study, the number of included patients was quite limited, despite a fair number of screened patients.

In conclusion, our data provide evidence that stopping or reducing the dose of ICS may be possible irrespective of baseline asthma control in a substantial part of non-eosinophilic asthmatics without compromising asthma control and the risk of exacerbations. This finding, which challenges the current international recommendations, urgently needs to be confirmed in large prospective multicentre randomized controlled trials.

\section{CONFLICT OF INTEREST}

SD, FS, VP, MH: none. TVH: grants from Amgen, outside the submitted work. RL: grants from GSK, Chiesi, Novartis and personal fees for advisory board and lectures from GSK, Chiesi, Novartis, Astra Zeneca, outside the submitted work.

\section{ORCID}

S. Demarche iD http://orcid.org/0000-0002-5628-0262

\section{REFERENCES}

1. Agache I, Akdis C, Jutel M, Virchow JC. Untangling asthma phenotypes and endotypes. Allergy. 2012;67:835-846.

2. Louis R, Schleich F, Barnes PJ. Corticosteroids: still at the frontline in asthma treatment? Clin Chest Med. 2012;33:531-541.

3. Global Initiative for Asthma (GINA). Global Strategy for Asthma Management and Prevention 2017.

4. Pavord ID. Non-eosinophilic asthma and the innate immune response. Thorax. 2007;62:193-194.

5. Berry M, Morgan A, Shaw DE, et al. Pathological features and inhaled corticosteroid response of eosinophilic and non-eosinophilic asthma. Thorax. 2007;62:1043-1049.

6. Pavord ID, Brightling CE, Woltmann G, Wardlaw AJ. Non-eosinophilic corticosteroid unresponsive asthma. Lancet. 1999;353:2213-2214.

7. Bacci $E$, Cianchetti S, Bartoli $M$, et al. Low sputum eosinophils predict the lack of response to beclomethasone in symptomatic asthmatic patients. Chest. 2006;129:565-572.

8. Cowan DC, Cowan JO, Palmay R, Williamson A, Taylor DR. Effects of steroid therapy on inflammatory cell subtypes in asthma. Thorax. 2010;65:384-390

9. Godon P, Boulet LP, Malo JL, Cartier A, Lemière C. Assessment and evaluation of symptomatic steroid-naive asthmatics without sputum eosinophilia and their response to inhaled corticosteroids. Eur Respir J. 2002;20:1364-1369.

10. Schleich FN, Manise M, Sele J, Henket M, Seidel L, Louis R. Distribution of sputum cellular phenotype in a large asthma cohort: predicting factors for eosinophilic vs neutrophilic inflammation. BMC Pulm Med. 2013;13:11.

11. Jatakanon A, Lim S, Barnes PJ. Changes in sputum eosinophils predict loss of asthma control. Am J Respir Crit Care Med. 2000;161:64 72.

12. Giannini D, Di Franco A, Cianchetti S, et al. Analysis of induced sputum before and after withdrawal of treatment with inhaled corticosteroids in asthmatic patients. Clin Exp Allergy. 2000;30:1777-1784.

13. Leuppi JD, Salome CM, Jenkins CR, et al. Predictive markers of asthma exacerbation during stepwise dose reduction of inhaled corticosteroids. Am J Respir Crit Care Med. 2001;163:406-412.

14. Deykin A, Lazarus SC, Fahy JV, et al. Sputum eosinophil counts predict asthma control after discontinuation of inhaled corticosteroids. J Allergy Clin Immunol. 2005;115:720-727.

15. Belda J, Parameswaran K, Lemière C, Kamada D, O'Byrne PM, Hargreave $\mathrm{FE}$. Predictors of loss of asthma control induced by corticosteroid withdrawal. Can Respir J. 2006;13:129-133. 
16. Green RH, Brightling CE, McKenna S, et al. Asthma exacerbations and sputum eosinophil counts: a randomised controlled trial. Lancet. 2002;360:1715-1721.

17. Schleich FN, Chevremont A, Paulus V, et al. Importance of concomitant local and systemic eosinophilia in uncontrolled asthma. Eur Respir J. 2014;44:97-108.

18. Demarche SF, Schleich FN, Paulus VA, Henket MA, Van Hees TJ, Louis RE. Asthma control and sputum eosinophils: a longitudinal study in daily practice. J Allergy Clin Immunol Pract. 2017;5:13351343. e5.

19. Nathan RA, Sorkness CA, Kosinski M, et al. Development of the asthma control test: a survey for assessing asthma control. J Allergy Clin Immunol. 2004;113:59-65.

20. Juniper EF, O'Byrne PM, Guyatt GH, Ferrie PJ, King DR. Development and validation of a questionnaire to measure asthma control. Eur Respir J. 1999;14:902-907.

21. Juniper EF, Guyatt GH, Cox FM, Ferrie PJ, King DR. Development and validation of the mini asthma quality of life questionnaire. Eur Respir J. 1999;14:32-38.

22. Foster JM, Schokker S, Sanderman R, Postma DS, van der Molen T. Development of a brief questionnaire (ICQ-S) to monitor inhaled corticosteroid side-effects in clinical practice. Allergy. 2014;69:372379.

23. Menckeberg TT, Bouvy ML, Bracke M, et al. Beliefs about medicines predict refill adherence to inhaled corticosteroids. J Psychosom Res. 2008;64:47-54.

24. Horne R, Weinman J. Patients' beliefs about prescribed medicines and their role in adherence to treatment in chronic physical illness. $J$ Psychosom Res. 1999;47:555-567.

25. Reddel HK, Taylor DR, Bateman ED, et al. An Official American Thoracic Society/European Respiratory Society Statement: asthma control and exacerbations: standardizing endpoints for clinical asthma trials and clinical practice. Am J Respir Crit Care Med. 2009;180:5999.

26. Lemière $\mathrm{C}$, Tremblay $\mathrm{C}$, FitzGerald $\mathrm{M}$, et al. Effects of a short course of inhaled corticosteroids in noneosinophilic asthmatic subjects. Can Respir J. 2011;18:278-282.

27. Demarche S, Schleich F, Henket M, Paulus V, Van Hees T, Louis R. Detailed analysis of sputum and systemic inflammation in asthma phenotypes: are paucigranulocytic asthmatics really non-inflammatory? BMC Pulm Med. 2016;16:46.

28. Chlumský J, Striz I, Terl M, Vondracek J. Strategy aimed at reduction of sputum eosinophils decreases exacerbation rate in patients with asthma. J Int Med Res. 2006;34:129-139.
29. Bacci E, Latorre $\mathrm{M}$, Cianchetti $\mathrm{S}$, et al. Transient sputum eosinophilia may occur over time in non-eosinophilic asthma and this is not prevented by salmeterol. Respirol Carlton Vic. 2012;17:11991206.

30. Petsky HL, Cates CJ, Lasserson TJ, et al. A systematic review and meta-analysis: tailoring asthma treatment on eosinophilic markers (exhaled nitric oxide or sputum eosinophils). Thorax. 2012;67:199208.

31. Haldar P, Pavord ID, Shaw DE, et al. Cluster analysis and clinical asthma phenotypes. Am J Respir Crit Care Med. 2008;178:218-224.

32. Rank MA, Hagan JB, Park MA, et al. The risk of asthma exacerbation after stopping low-dose inhaled corticosteroids: a systematic review and meta-analysis of randomized controlled trials. J Allergy Clin Immunol. 2013;131:724-729.

33. Hagan JB, Samant SA, Volcheck GW, et al. The risk of asthma exacerbation after reducing inhaled corticosteroids: a systematic review and meta-analysis of randomized controlled trials. Allergy. 2014;69:510-516.

34. Crossingham I, Evans DJ, Halcovitch NR, Marsden PA. Stepping down the dose of inhaled corticosteroids for adults with asthma. Cochrane Database Syst Rev. 2017; 2: CD011802.

35. Buhl R. Local oropharyngeal side effects of inhaled corticosteroids in patients with asthma. Allergy. 2006;61:518-526.

36. Raissy HH, Kelly HW, Harkins M, Szefler SJ. Inhaled corticosteroids in lung diseases. Am J Respir Crit Care Med. 2013;187:798-803.

37. Louis R, Demarche S. Time for reasoning ICS prescription in obstructive airway diseases. Int J Clin Pract. 2014;68:1176-1178.

38. McKeever T, Harrison TW, Hubbard R, Shaw D. Inhaled corticosteroids and the risk of pneumonia in people with asthma: a casecontrol study. Chest. 2013;144:1788-1794.

39. McGrath KW, Icitovic N, Boushey HA, et al. A large subgroup of mild-to-moderate asthma is persistently noneosinophilic. Am J Respir Crit Care Med. 2012;185:612-619.

How to cite this article: Demarche S, Schleich F, Henket M,

Paulus V, Louis R, Van Hees T. Step-down of inhaled corticosteroids in non-eosinophilic asthma: A prospective trial in real life. Clin Exp Allergy. 2018;48:525-535.

https://doi.org/10.1111/cea.13106 